\title{
SENSITIVITY OF RESONANT TUNNELING DIODE PHOTODETECTORS
}

\author{
Andreas Pfenning, ${ }^{1, a)}$ Fabian Hartmann, ${ }^{1}$ Fabian Langer, ${ }^{1}$ Martin Kamp, ${ }^{1}$ Sven Höfling, ${ }^{1,2}$ \\ Lukas Worschech ${ }^{1}$ \\ 1) Technische Physik and Wilhelm-Conrad-Röntgen-Research Center for Complex Material Systems, \\ Physikalisches Institut, Universität Würzburg, Am Hubland, D-97074 Würzburg, Germany \\ 2) SUPA, School of Physics and Astronomy, University of St. Andrews, St. Andrews, KY16 9SS, United Kingdom
}

We have studied the sensitivity of $\mathrm{AlGaAs} / \mathrm{GaAs}$ double barrier resonant tunneling diode photodetectors with an integrated GaInNAs absorption layer for light sensing at the telecommunication wavelength of $\lambda=$ $1.3 \mu \mathrm{m}$ for illumination powers from pico to micro Watts. The sensitivity decreases nonlinearly with power. An illumination power increase of seven orders of magnitude leads to a reduction of the photocurrent sensitivity from $S_{I}=5.82 \times 10^{3} \mathrm{~A} / \mathrm{W}$ to $3.2 \mathrm{~A} / \mathrm{W}$. We attribute the nonlinear sensitivity-power dependence to an altered local electrostatic potential due to hole-accumulation that on the one hand tunes the tunneling current, but on the other hand affects the lifetime of photogenerated holes. In particular, the lifetime decreases exponentially with increasing hole-population. The lifetime reduction results from an enhanced electrical field, a rise of the quasi-Fermi level and an increased energy splitting within the triangular potential well. The non-constant sensitivity is a direct result of the non-constant lifetime. Based on these findings, we provide an expression that allows to calculate the sensitivity as a function of illumination power and bias voltage, show a way to model the time-resolved photocurrent, and determine the critical power up to which the resonant tunneling diode photosensor sensitivity can be assumed constant.

a) Electronic mail to: Andreas.Pfenning@physik.uni-wuerzburg.de 


\section{INTRODUCTION}

During the past few years, resonant tunneling diodes (RTDs) ${ }^{1-3}$ have been increasingly deployed and investigated as sensors for various physical quantities, such as temperature, ${ }^{4}$ pressure, ${ }^{5}$ or light. ${ }^{6}$ Of particular interest are RTDs with embedded quantum dots operated as single photon detectors and photon counters, ${ }^{7-10}$ or high-gain RTD photodetectors for room temperature telecommunication wavelength light sensing. ${ }^{11}$ Hereby, the RTD serves as an internal amplifier of weak electric signals, caused by photogenerated charge carriers. ${ }^{12}$ The high internal amplification, low-voltage operation, and supplementary functionality, inherently provided by the region of negative differential conductance (NDC), qualify RTD photodetectors as a good alternative to avalanche photodiodes (APDs). Similar to APDs, the RTD device performance is tuned by the applied bias voltage. In a recent publication, ${ }^{13}$ we found that the RTD photocurrent-voltage relation is a nonlinear function, which can be described by three voltagedependent parameters: the RTD current-voltage characteristics in the dark $I_{\text {dark }}(V)$, the RTD quantum efficiency $\eta(V)$, and the mean lifetime $\tau(V)$ of photogenerated holes accumulated in vicinity to the resonant tunneling structure. Furthermore, the RTD photocurrent-voltage relation is not only a nonlinear function of the applied bias voltage, but also a nonlinear function of the incident illumination power $P .{ }^{11,14}$ Only for weak illumination powers, a constant sensitivity is observed and the RTD photocurrent can be approximated as a linear function of $P .{ }^{11,15}$ The linear approximation for weak illumination powers might serve well enough for RTDs operated as photon counters, yet it does not suffice for RTD photodetectors exposed to a wide range of illumination powers across several orders of magnitude. England et al. were the first to qualitatively name and describe the sources of the nonlinear sensitivity, such as a decreasing lifetime of accumulated holes, due to increased voltage drop across the resonant tunneling structure (RTS) and a higher quasi-Fermi level. ${ }^{14}$ Later in a study by Dong et al., ${ }^{16}$ the nonlinear sensitivity was ascribed to a repulsive interaction between already accumulated holes at the RTS and newly photogenerated holes.

In this work, we study the sensitivity of RTD photodetectors for light sensing at the telecommunication wavelength of $\lambda=1.3 \mu \mathrm{m}$ across a broad range of illumination powers from $P=2.7 \mathrm{pW}$ up to $P=18.7$ $\mu \mathrm{W}$, thus covering seven orders of magnitude. A non-constant sensitivity was found, and is attributed to a variation of the local electrostatic potential under illumination due to photogenerated minority charge carriers, which alters the voltage-dependent parameters that govern the RTD hole accumulation dynamics. We find that due to the enhanced electrical field across the RTS, the lifetime of photogenerated holes decreases exponentially with an increasing hole-population. Combined with our previous findings on the photocurrent-voltage relation, we are able to describe the sensitivity of RTD photodetectors as a function of bias voltage and illumination power, and provide a critical power $P_{\text {crit }}$ up to which the sensitivity can be assumed constant.

\section{METHODS AND FABRICATION}


Figure 1 provides detailed information on sample design and working principle. A three dimensional color-coded sketch of the RTD photodetector is shown in Figure 1 (a). The studied RTD was grown by molecular beam epitaxy and is based on an $\mathrm{Al}_{0.6} \mathrm{Ga}_{0.4} \mathrm{As} / \mathrm{GaAs}$ double barrier resonant tunneling structure (AlGaAs: green \& GaAs: grey) with a nearby and lattice-matched $\underline{\text { Ga }} \underline{0.89} \underline{\mathbf{n}_{0.11}} \underline{\mathrm{N}_{0.04}} \underline{\mathrm{s}_{0.96}} \underline{\text { absorption layer }}$ (blue) for light sensing at the telecommunication wavelength at $\lambda=1.3 \mu \mathrm{m}$. Circular RTD mesa structures were defined by electron beam lithography and dry-chemical etching. The studied sample has a mesa diameter of $5 \mu \mathrm{m}$. An Au ring-shaped contact on top of the RTD mesa provides optical access and electrical contact. The second contact was alloyed on the backside of the substrate. Further information on sample design and fabrication, e.g. mole fraction and growth condition of the GalnNAs absorption layer, is given in Refs. $[13,15]$. The schematic conduction (CB) and valence band (VB) profile under an applied bias voltage $V$ is shown in Figure 1(b). Incident photons excite electron-hole pairs in the GalnNAs absorption layer. Within the external electrical field, photogenerated holes $\left(\mathrm{h}^{+}\right)$drift towards the resonant tunneling structure (RTS) where they accumulate. The accumulated positive charge induces a variation of the local electrostatic field and causes an additional voltage drop across the RTS, which in turn modulates the resonant tunneling current of electrons $\left(\mathrm{e}^{-}\right)$. For the opposite bias voltage direction, no hole accumulation occurs and hence the photocurrent vanishes almost completely. ${ }^{13}$ Although, a photocurrent might emerge at large illumination powers due to an enhanced photoconductivity, even when hole accumulation is absent. ${ }^{17}$ At low illumination powers however, charge accumulation is the dominant effect, while the enhanced photoconductivity is negligible small. ${ }^{17,18}$ In our sensor and for a wavelength of $\lambda=1.3 \mu \mathrm{m}$, absorption occurs only within the GalnNAs region. Therefore, a positive bias voltage needs to be applied to the Au ring-shaped contact, which guarantees charge accumulation at the RTS. A detailed description and analysis of the photocurrent-voltage relation of RTD photodetectors is provided in Ref. [13].

The RTD was investigated by means of electro-optical transport measurements, operated in a twoterminal setup. A Hewlett-Packard 3251 high-precision voltage source was used to apply a DC bias voltage $V_{\text {in }}$ to the Au ring-shaped contact, while the backside contact (substrate) was connected to the ground (GND) via an Ohmic resistor $R$. The electrical read-out was done by measuring the voltage drop $V_{\text {out }}$ across $R=1.00 \mathrm{k} \Omega$ with a digital Keithley 200 Multimeter (in case of DC measurements), or with a digital oscilloscope (in case of time-resolved measurements). The bias voltage across the RTD is given by $V=V_{\text {in }}-$ $V_{\text {out }}$, and the current is $I=V_{\text {out }} / R$. A laser with emission wavelength of $\lambda=1.3 \mu \mathrm{m}$ was used for illumination and focused on the RTD mesa by a 50-X near infrared microscope objective. All experiments were conducted at room temperature.

\section{EXPERIMENT, RESULTS AND DISCUSSION}


Figure 2 (a) shows the current-voltage (I(V)-) characteristics measured in the dark (solid black line) and under illumination (solid red line). The I(V)-characteristics is bistable with a resonance current of $I_{\text {res }}=$ $1.38 \mathrm{~mA}$ and a valley current of $I_{v a l}=0.66 \mathrm{~mA}$ is observed. Under illumination (solid red line), photogenerated holes accumulate in vicinity of the RTS causing the I(V)-characteristic to shift towards smaller voltages caused by the photoinduced voltage shift $\Delta V \cdot{ }^{13,17,18}$ The photocurrent $I_{P h}=I_{i l l u}-I_{d a r k}$ is obtained by subtracting the current in the dark $\left(I_{\text {dark }}\right)$ from the current under illumination $\left(I_{\text {illu }}\right)$. For negative bias voltages, i.e. when a negative bias voltage is applied to the Au ring-shaped contact, no hole accumulation at the RTS takes place. As a result, no photocurrent can be observed. ${ }^{11,13}$

The photocurrent $I_{P h}$ was measured as a function of illumination power $P$ across a range of seven orders of magnitude from $P=2.7 \mathrm{pW}$ up to $P=18.7 \mu \mathrm{W}$, as is shown in Figure 2(b). The RTD was biased at $V=1.90 \mathrm{~V}$, which had been identified to be the optimal working point in terms of maximum photocurrent. ${ }^{13}$ In consistence with previous results, ${ }^{11,15}$ a monotonically yet nonlinear increase of $I_{P h}$ with $P$ is observed. The increase of $I_{P h}$ flattens and saturates at large $P$. Only in case of low illumination powers $P \lesssim 40 \mathrm{pW}$ (see Figure $2(\mathrm{~d})$ ), a linear approximation is valid and a constant small signal photocurrent sensitivity $S_{I}=\Delta I_{P h} / \Delta P=(5.82 \pm 0.20) \times 10^{3} \mathrm{~A} / \mathrm{W}$ can be extracted from the best linear fit. From $S_{I}$, the spectral response $R_{\lambda}=1.05 \mathrm{~A} / \mathrm{W}$ at $\lambda=1.30 \mu \mathrm{m}$, and the RTD quantum efficiency of $\eta=1.68 \%$, ${ }^{13}$ the internal multiplication factor is estimated to be $M=S /\left(\eta R_{\lambda}\right)=(3.30 \pm 0.11) \times 10^{5}$. Thus, each absorbed photon triggers an average of 330000 excess electrons. Due to the flattening increase of $I_{P h}(P)$ at larger powers, the multiplication factor declines down to $M \approx 181$ at $P=18.7 \mu \mathrm{W}$. Usually to evaluate photodetectors, the figure of merit (beside sensitivity) is the noise-equivalent power (NEP). From the experimentally determined value of $S_{I}$ and the theoretical shot noise $i_{\text {shot }}=\sqrt{2 \mathrm{q}_{0} \mathrm{I}^{2}}=1.23 \times$ $10^{-12} \mathrm{~A} / \mathrm{Hz}^{1 / 2}$, the noise-equivalent power of the presented device is calculated to be $\mathrm{NEP}=$ $\mathrm{i}_{\text {shot }} / \mathrm{S}_{\mathrm{I}}=(2.10 \pm 0.07) \times 10^{-16} \mathrm{~W} / \mathrm{Hz}^{1 / 2}$. This NEP value represents the ideal and lowest possible value. Noise measurements in similar RTD photodetectors however indicate, that the actual noise value is about a factor of about 30 larger compared to the theoretical shot noise limit, ${ }^{19}$ which would yield larger and hence worse NEPs. Though, there are many intertwining factors contributing to the noise in RTD photodetectors, which need to be further investigated.

To analyze the RTD photodetectors in detail, the photoinduced voltage shift $\Delta V$ of the I(V)characteristics under illumination must be considered. $\Delta V$ sheds light on the population of accumulated holes at the RTS and is what technically causes the photocurrent. As indicated in Figure 2(a), $\Delta V$ and $I_{P h}$ are inseparable linked via: ${ }^{13,17}$

$$
I_{P h}(V)=I_{\text {dark }}(V+\Delta V)-I_{\text {dark }}(V),
$$

with $I_{\text {illu }}(V)=I_{\text {dark }}(V+\Delta V)$. Figure 2 (c) displays $\Delta V$ as a function of illumination power. Similar to the photocurrent, $\Delta V$ increases monotonically yet nonlinearly and flattens for large $P$. For low illumination 
powers ( $P \lesssim 40 \mathrm{pW}$, see Figure $2(\mathrm{e})$ ), $\Delta V$ increases linearly with $P$ and from the best linear fit, a constant voltage shift sensitivity of $S_{V}=(4.32 \pm 0.15) \times 10^{8} \mathrm{~V} / \mathrm{W}$ was extracted.

\section{Lifetime-Reduction}

In Figure $3(\mathrm{a}), S_{V}(P)=\Delta V(P) / P$ is shown as a function of $P$. Experimental values are depicted as black spheres, while the constant small signal sensitivity from Figure 2 (e) is indicated by the solid red line. The sensitivity decreases monotonically by four orders of magnitude down to $S_{V}=6.6 \times 10^{4} \mathrm{~V} / \mathrm{W}$ at $P=18.7$ $\mu \mathrm{W}$, which is due to the flattening of $\Delta V$ with increasing $P$ (see Figure 2(c)).

We attribute the non-constant and decreasing sensitivity to hole-accumulation at the RTS being a selflimiting process. ${ }^{14}$ Several intertwining factors are working together in limiting $\Delta V$ for increasing powers. As shown by Coêlho et al., the photoinduced voltage-shift $\Delta V$ is proportional to the number of accumulated holes $N_{h}$ and given by: ${ }^{13,17}$

$$
\Delta V=N_{h} \times \frac{V_{h}}{n_{R T D}}
$$

The RTD leverage factor $n_{R T D}$ can be extracted from the I(V)-characteristics in the dark, ${ }^{20}$ whereas $V_{h}$ (additional voltage drop across RTS caused by a single hole) can be calculated by a simple electrostatic model. ${ }^{17}$ In a previous publication, we have identified $n_{R T D}=(2.6 \pm 0.3) \%$ and $V_{h}=(0.89 \pm 0.05) \mu \mathrm{V} .{ }^{13}$ The number of accumulated holes at the RTS is governed by a simple rate-equation: ${ }^{14}$

$$
\frac{\mathrm{d}}{d t} N_{h}=-\frac{1}{\tau} N_{h}+\eta \frac{P}{h v}
$$

The first term at the right hand side describes the rate of escape (see Figure 1 (b)). It is quantified by the mean lifetime of accumulated holes $\tau$. The second term describes the rate of incoming photogenerated holes (see Figure 1 (b)). It is quantified by the product of the incident photon flux $P / h v$ (with the photon energy $h v$ ) and the RTD quantum efficiency $\eta$. The RTD quantum efficiency is defined as external quantum efficiency, i.e. the probability of an incident photon to excite a minority charge carrier (hole) that is captured for accumulation at the RTS. Solving Eq. (3) for $N_{h}$ under cw-illumination and equilibrium condition $\left(d N_{h} / d t=0\right)$ and inserting $N_{h}$ into Eq. (2) yields a linear relation between $\Delta V$ and $P$ :

$$
\Delta V(P)=\tau \eta \frac{1}{h v} \frac{V_{h}}{n_{R T D}} \times P
$$

and thus a constant voltage shift sensitivity independent of $P$ :

$$
\mathrm{S}_{\mathrm{V}}=\tau \eta \frac{1}{h v} \frac{V_{h}}{n_{R T D}}
$$

With the experimentally determined values from our previously published time-resolved photocurrent measurements ${ }^{13}$ of $\tau=116 \mu \mathrm{s}$ and $\eta=1.68 \%$ at $V=1.90 \mathrm{~V}$, the small signal voltage shift sensitivity after 
Eq. (5) is calculated to be $S_{V}=(4.48 \pm 0.60) \times 10^{8} \mathrm{~V} / \mathrm{W}$. This is in excellent agreement with the value of $S_{V}=(4.32 \pm 0.15) \times 10^{8} \mathrm{~V} / \mathrm{W}$ extracted from the best linear fit (see Figure $\left.2(\mathrm{e})\right)$.

Above $P \gtrsim 40 \mathrm{pW}$ (see Fig 3(a), black spheres), the sensitivity declines with increasing $P$ and hence, hole-accumulation is strong enough to affect the voltage-dependent parameters, $\tau(V)$ and $\eta(V)$, governing the hole-accumulation dynamics. Both RTD quantum efficiency and mean lifetime of accumulated holes were found to be voltage dependent. ${ }^{13}$ These dependencies must be taken into account, when solving Eq. (3). At $V=1.90 \mathrm{~V}, \eta(V)$ is constant with a value of $\eta_{0}=1.68 \%$, leaving $\tau(V)$ as only parameter. $\tau(V)$ decreases exponentially with $V$, as shown in Figure $3(b)$ :

$$
\tau(V)=\tau_{0} \exp (-\alpha V)
$$

$\tau_{0}$ is the projected lifetime at zero bias voltage and $\alpha$ is the decay parameter. Figure 3 (b) depicts experimentally determined values of $\tau$ as a function of $V$ at small illumination powers (black spheres). At $V=1.90 \mathrm{~V}, \tau=116 \mu$ s and $\alpha=2.69 \mathrm{~V}^{-1}$. A large accumulation of photogenerated holes at the RTS leads to the photoinduced voltage shift $\Delta V$, which in turn changes the transmission probability of electrons and hence the resonant tunneling current. But the additional voltage drop also leads to a reduction of the mean lifetime of accumulated holes (see inset in Figure 3 (a)). To describe the reduction of mean lifetime due to $\Delta V$, we insert $\Delta V$ into the exponential decay equation Eq. (6):

$$
\tau(V+\Delta V)=\tau_{0} \exp (-\alpha(V+\Delta V))
$$

In order to solve the rate equation (Eq. (3)) describing the hole-accumulation dynamics, we use Eq. (2) and rewrite Eq. (7) as a function of the number of accumulated holes:

$$
\tau(V, N)=\tau_{0} \exp \left(-\alpha\left(V+\mathrm{N}_{\mathrm{h}} \frac{V_{h}}{n_{R T D}}\right)\right) .
$$

Eq. (8) can be further simplified, and the voltage-dependent and hole-accumulation dependent parts can be separated:

$$
\begin{gathered}
\tau(V, N)=\tau_{0} \exp (-\alpha V) \exp \left(-\alpha \frac{V_{h}}{n_{R T D}} N_{h}\right) \\
\tau(V, N)=\tau(V) \times \exp \left(-\zeta N_{h}\right) .
\end{gathered}
$$

With $\tau(V)$ being the small signal mean lifetime of holes accumulated at the RTS for a bias voltage $V$, and $\exp \left(-\zeta N_{h}\right)$ as the lifetime-reduction due to hole-accumulation of a number of $N_{h}$ holes. The decay parameter $\zeta$ is given by:

$$
\zeta=-\alpha \beta \frac{V_{h}}{n_{R T D}}
$$


In case the additional voltage drop across the RTS is the only lifetime-reducing factor, $\beta=1$ and hence $\zeta=(93 \pm 11) \times 10^{-6}$. However as England et. al. pointed out, ${ }^{14}$ further lifetime-reducing factors should be considered, such as an increasing quasi-Fermi level, or the enhanced energy splitting of confined hole states due to the enhanced electric field. To account for these additional lifetime reductions, we introduced $\beta$ as an extra empirical parameter, which we expect to be $\beta \geq 1$. To calculate an expression for $\Delta V(P)$, we insert Eq. (10) into Eq. (3) and solve for $N_{h}(P)$ for equilibrium conditions $\left(d N_{h} / d t=0\right)$. The equation can be solved analytically and we find, that $\Delta V(P)$ follows the Lambert-W function (also known as ProductLogarithm):

$$
\Delta V(V, P)=\frac{V_{h}}{\zeta n_{R T D}} \times \operatorname{LambertW}\left(\tau(V) \eta(V) \zeta \frac{P}{h v}\right) .
$$

The voltage shift sensitivity is given by:

$$
\mathrm{S}_{\mathrm{V}}(V, P)=\frac{1}{P} \frac{V_{h}}{\zeta n_{R T D}} \times \operatorname{LambertW}\left(\tau(V) \eta(V) \zeta \frac{P}{h v}\right) .
$$

We use Eq. (13) to fit $S_{V}(P)$ with $\zeta$ being the only fitting parameter. The resulting best fit (solid green line) is shown in Figure 3 (a), and shows an excellent agreement with the experimental data across the complete measured power range from $P=2.7 \mathrm{pW}$ up to $P=18.7 \mu \mathrm{W}$. We find that $\zeta=(273 \pm 5) \times$ $10^{-6}$ and hence, $\beta=(2.94 \pm 0.12)$, which verifies that several different factors contribute to a reduction of the mean lifetime of accumulated holes when the hole-population is increased. Figure 3 (c) shows the lifetime reduction for $\beta=1$ (only $\Delta V$ is considered; dark green spheres), and when $\beta=(2.94 \pm 0.12)$ (experimentally determined value, black spheres). As long as the number of accumulated holes is low, $\tau$ is unchanged. However at about $N_{h} \approx 10^{4}$, the mean lifetime is reduced to almost $1 / 500$ of its small signal value.

The reduction of the mean lifetime with increasing $P$ can indeed be observed in time-resolved photocurrent measurements. The RTD was excited with rectangular laser pulses of $100 \mu$ sidth and the photocurrent was measured as a function of time for two different on-illumination powers $P=120 \mathrm{pW}$ and $P=24 \mathrm{nW}$. Figure $3(\mathrm{~d})$ shows the two normalized time traces of the photocurrent. The photocurrent rises when the laser is switched on (at $t=100 \mu \mathrm{s}$ ). When the laser is switched off (at $t=200 \mu \mathrm{s}$ ), the photocurrent decreases down to zero. The off/on and on/off time scale at high illumination power occurs much faster compared to low illumination power. We exemplarily used the input parameters from the experiment and Eq. (1), (2), (3) and (10) to numerically calculate the time resolved photocurrent for both on-illumination powers of $P=24 \mathrm{nW}$ and $P=120 \mathrm{pW}$. The resulting time-traces are shown in Figure 3 (e) as black and red line, respectively. Indeed, the calculated time traces are in good agreement with the experimental ones, demonstrating that the mean lifetime is reduced for increasing illumination powers, due to an increased voltage drop across the RTS and quasi-Fermi level shift. ${ }^{14}$ 


\section{Critical Illumination Power}

For the operation of sensors, it is most convenient when the sensitivity is flat and constant and the output is a linear function of the input. For RTD photodetectors this is the case only for small illumination powers, hence it is crucial to know up to which critical power $P_{\text {crit }}$ the RTD photosensitivity can be assumed flat and constant. From Eq. (5) and Eq. (13) we can calculate $P_{\text {crit }}$ :

$$
P_{c r i t}(V)=\frac{h v}{\eta(V) \tau(V) \zeta} \times \frac{1}{1-c} \ln \left(\frac{1}{1-c}\right) .
$$

$\tau(V)$ is determined by Eq. (6), and $\eta(V)$ is the RTD quantum efficiency. $\eta(V)$ is voltage dependent and follows an error-function, which reflects that a critical threshold voltage $V_{t h}$ must be applied to overcome the built-in electrical field and ensure efficient capturing of photogenerated holes at the RTS: ${ }^{13}$

$$
\eta(V)=\eta_{0}\left(1+\operatorname{erf}\left(\gamma\left(V-V_{t h}\right)\right)\right) / 2
$$

Below and well above $V_{t h}, \eta(V)$ approaches zero and a constant value of $\eta_{0}$, respectively. $\eta_{0}$ accounts for absorption within the GalnNAs layer and loss mechanisms such as reflections at the semiconductor air interface or radiative and non-radiative recombination. $\gamma$ is a parameter that accounts for the steepness of the transition. The parameter $c$ is defined as a deviation parameter between 0 and 1 . In case $S_{V}(P)$ has decreased down to $99 \%$ of the constant small signal sensitivity, the deviation is $1 \%$, and hence $c=0.01$. In our case, we define the critical $P_{\text {crit }}$ for a deviation of less then $10 \%$, and hence $c=0.1$ :

$$
P_{c r i t}(V)=\frac{h v}{\eta(V) \tau(V) \zeta} \times 0.117
$$

Figure 4 shows $P_{\text {crit }}(V)$, the normalized small signal photocurrent $S_{I}(V) / S_{I, \max }$ and the voltage shift sensitivity $S_{V}(V) / S_{V, \max }$ as a function of bias voltage. The photocurrent sensitivity is shown as blue stars (experiment) and solid blue line (model), whereas the voltage shift sensitivity is shown as dark green spheres (experiment) and solid dark green line (model). As one can see from Eq. (16), the $P_{\text {crit }}$ follows the inverse product of the RTD quantum efficiency and the mean lifetime of accumulated holes. $P_{\text {crit }}$ has a minimum at $V=1.32 \mathrm{~V}$. At this voltage, hole accumulation is most efficient and $S_{V}(V)$ and the product of $\eta(V)$ and $\tau(V)$ are maximal. The highest photocurrent sensitivity can be found at $V=1.90 \mathrm{~V}$, which is the operation voltage at which the power-dependent photocurrent measurement has been performed. At $V=$ $1.90 \mathrm{~V}$, the critical power has increased up to a value of $P_{\text {crit }}=(34 \pm 4) \mathrm{pW}$. Since $\mathrm{P}_{\text {crit }}$ is proportional to

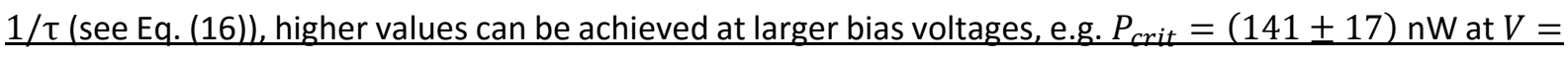
$5.00 \mathrm{~V}$, which is one method to increase the range of flat and constant sensitivity. A second method to achieve larger $\mathrm{P}_{c r i t}$ would be to optimize the heterostructure design layout to reduce $\tau$, for example via a reduction of the barrier width or height of the valence band. 


\section{Quantum Efficiency Threshold Shift}

So far, we have investigated the effect of an increasing hole-accumulation on the mean lifetime of accumulated holes. By operating the RTD at a large enough bias voltage, we ensured a constant quantum efficiency independent of $N_{h}$. This assumption is not valid for smaller bias voltages. To identify the effect of hole accumulation on the RTD quantum efficiency $\eta$, we measured the photoinduced voltage shift $\Delta V(V)$ as a function of bias voltage $V$ from $V=0$ to $5 \mathrm{~V}$ for illumination powers from $P=8.6 \mathrm{nW}$ to $2.8 \mu \mathrm{W}$. Figure 5 (a) shows $\Delta V(V)$ for $P=2.8 \mu \mathrm{W}$ (black spheres). For $V \leq 0.5 \mathrm{~V}$, the current is below the resolution limit of our setup, thus no experimental data of $\Delta V$ could be provided in this region. At about $V=1.6 \mathrm{~V}, \Delta V$ shows a maximum of about $0.9 \mathrm{~V}$. On the high-voltage side, $\Delta V$ is limited by a decreasing lifetime $\tau$, as discussed previously. On the low-voltage side however, $\Delta V$ is limited by the RTD quantum efficiency $\eta$. Figure 5 (a) displays the theoretical characteristic of $\Delta V(V)$ (solid red line) after Eq. (12) with $\tau(V)$ from Eq. (6) and $\eta(V)$ from Eq. (15), where the small signal threshold voltage $V_{t h}=1.22 \mathrm{~V}$ was assumed. ${ }^{13}$ Theory and experiment show an excellent agreement for $V \geq 1.75 \mathrm{~V}$ but a significant deviation for smaller bias voltages. A best fit after Eq. (12), with $V_{t h}$ as only fitting parameter (green dashed line), indicates that $V_{t h}$ has shifted up to $V_{t h}=1.70 \mathrm{~V}$ under illumination.

We have determined $V_{t h}$ for the remaining illumination powers of $P=0.32 \mu \mathrm{W}, 49 \mathrm{nW}$ and $8.4 \mathrm{nW}$. $\eta(V)$ is depicted as a function of $V$ for the remaining illumination powers. $\eta_{0}$ does not change under illumination. For high illumination powers, a large accumulation of positive charge induces an opposite electric field and $V_{t h}$ shifts towards higher voltages when the hole-density is increased. $V_{t h}$ is shown in the inset in Figure 4 (b) as a function of $P$ on the logarithmic scale. A well pronounced and linear shift of $V_{t h}$ to higher voltages with increasing $P$ can be observed. Having identified the effect of $N_{h}$ on $\eta(V)$ and $\tau(V)$, the photoinduced voltage shift $\Delta V$ can be modeled as a function of $P$ and $V$. Therefore, we insert $\tau(V)$ as given by Eq. (6) and $\eta(V)$ as given by Eq. (15) into Eq. (12), and include the power-dependence of $V_{t h}$. A comparison between experiment and model is given in Figure 6, which shows two colored contour plots of the photoinduced voltage shift $\Delta V$ as a function of $V$ and $P$. Orange represents the highest measured voltage shift of $\Delta V=0.9 \mathrm{~V}$ and blue zero voltage shift. Again, an excellent agreement between experiment (left-hand side) and simulation (right-hand side) is found.

\section{CONCLUSIONS}

In summary, we have presented a general study on the sensitivity of RTD photodetectors. The sensitivity of RTD photodetectors is not constant and strongly depends on the incident illumination power and bias voltage. While accumulation of photogenerated holes at the resonant tunneling structure is the foundation of RTD photoresponse and its high carrier multiplication, it also affects internal parameters that govern the charge carrier accumulation processes. The most influential parameter is the mean lifetime of accumulated holes, which decreases exponentially with increasing population of accumulated holes. When the number 
of holes increases, so does the voltage drop across the RTS, the quasi-Fermi level and the splitting between the triangular hole-states, which all contributes to a decreasing lifetime. In contrast, the RTD quantum efficiency can be assumed constant, except in cases when the RTD is biased close to the quantum efficiency threshold voltage, which slightly shifts towards larger voltages. The RTD photoresponse can then be described by a simple rate equation with non-constant lifetime. For $\mathrm{cw}$-illumination the rate equation can be solved analytically. The resulting Lambert-W function describes the photoinduced voltage shift as a function of illumination power and bias voltage, and is valid across the complete studied power and bias voltage range.

\section{ACKNOWLEDGEMENTS}

The authors are grateful for financial support by the BMBF via national project EIPHRIK (FKZ: 13N10710) and the European Union (FPVII (2007-2013) under Grant Agreement No. 318287 LANDAUER). The expert technical assistance by M. Emmerling and S. Handel is gratefully acknowledged. 


\section{REFERENCES}

${ }^{1}$ R. Tsu and L. Esaki, Appl. Phys. Lett. 22, 562 (1973).

2 L.L. Chang, L. Esaki, and R. Tsu, Appl. Phys. Lett. 24, 593 (1974).

${ }^{3}$ G. Haddad, P. Mazumder, and J.N. Schulman, Proc. IEEE 86, 641 (1998).

${ }^{4}$ A. Pfenning, F. Hartmann, M. Rebello Sousa Dias, L.K. Castelano, C. Süßmeier, F. Langer, S. Höfling, M. Kamp, G.E. Marques, L. Worschech, and V. Lopez-Richard, ACS Nano 9, 6271 (2015).

${ }^{5}$ J. Li, H. Guo, J. Liu, J. Tang, H. Ni, Y. Shi, C. Xue, Z. Niu, W. Zhang, M. Li, and Y. Yu, Nanoscale Res. Lett. 8, 218 (2013).

${ }^{6}$ B. Romeira, L.M. Pessoa, H.M. Salgado, C.N. Ironside, and J.M.L. Figueiredo, Sensors (Basel). 13, 9464 (2013).

7 J. Blakesley, P. See, A. Shields, B. Kardynał, P. Atkinson, I. Farrer, and D. Ritchie, Phys. Rev. Lett. 94, 067401 (2005).

${ }^{8}$ H.W. Li, B.E. Kardynał, P. See, A. J. Shields, P. Simmonds, H.E. Beere, and D. A. Ritchie, Appl. Phys. Lett. 91, 073516 (2007).

${ }^{9}$ R.H. Hadfield, Nat. Photonics 3, 696 (2009).

${ }^{10}$ Q. Weng, Z. An, B. Zhang, P. Chen, X. Chen, Z. Zhu, and W. Lu, Sci. Rep. 5, 9389 (2015).

${ }^{11}$ A. Pfenning, F. Hartmann, F. Langer, S. Höfling, M. Kamp, and L. Worschech, Appl. Phys. Lett. 104, 101109 (2014).

${ }^{12}$ W. Wang, Y. Hou, D. Xiong, N. Li, W. Lu, W. Wang, H. Chen, J. Zhou, E. Wu, and H. Zeng, Appl. Phys. Lett. 92, 023508 (2008).

${ }^{13}$ A. Pfenning, F. Hartmann, M. Rebello Sousa Dias, F. Langer, M. Kamp, L.K. Castelano, V. Lopez-Richard, G.E. Marques, S. Höfling, and L. Worschech, Appl. Phys. Lett. 107, 081104 (2015).

${ }^{14}$ P. England, J.E. Golub, L.T. Florez, and J.P. Harbison, Appl. Phys. Lett. 58, 887 (1991).

${ }^{15}$ F. Hartmann, F. Langer, D. Bisping, A. Musterer, S. Höfling, M. Kamp, A. Forchel, and L. Worschech, Appl. Phys. Lett. 100, 172113 (2012).

${ }^{16}$ Y. Dong, G. Wang, H. Ni, J. Chen, F. Gao, B. Li, K. Pei, and Z. Niu, Opt. Commun. 355, 274 (2015).

${ }^{17}$ I.J.S. Coêlho, J.F. Martins-Filho, J.M.L. Figueiredo, and C.N. Ironside, J. Appl. Phys. 95, 8258 (2004).

18 P.W. Park, H.Y. Chu, S.G. Han, Y.W. Choi, G. Kim, and E.-H. Lee, Appl. Phys. Lett. 67, 1241 (1995).

${ }^{19}$ F. Hartmann, F. Langer, D. Bisping, A. Musterer, S. Höfling, M. Kamp, A. Forchel, and L. Worschech, Proc. SPIE 8511, Infrared Remote Sensing and Instrumentation XX, 85110G (October 24, 2012)

${ }^{20}$ J. Schulman and H.D.L. Santos, Electron Device Lett. (1996). 

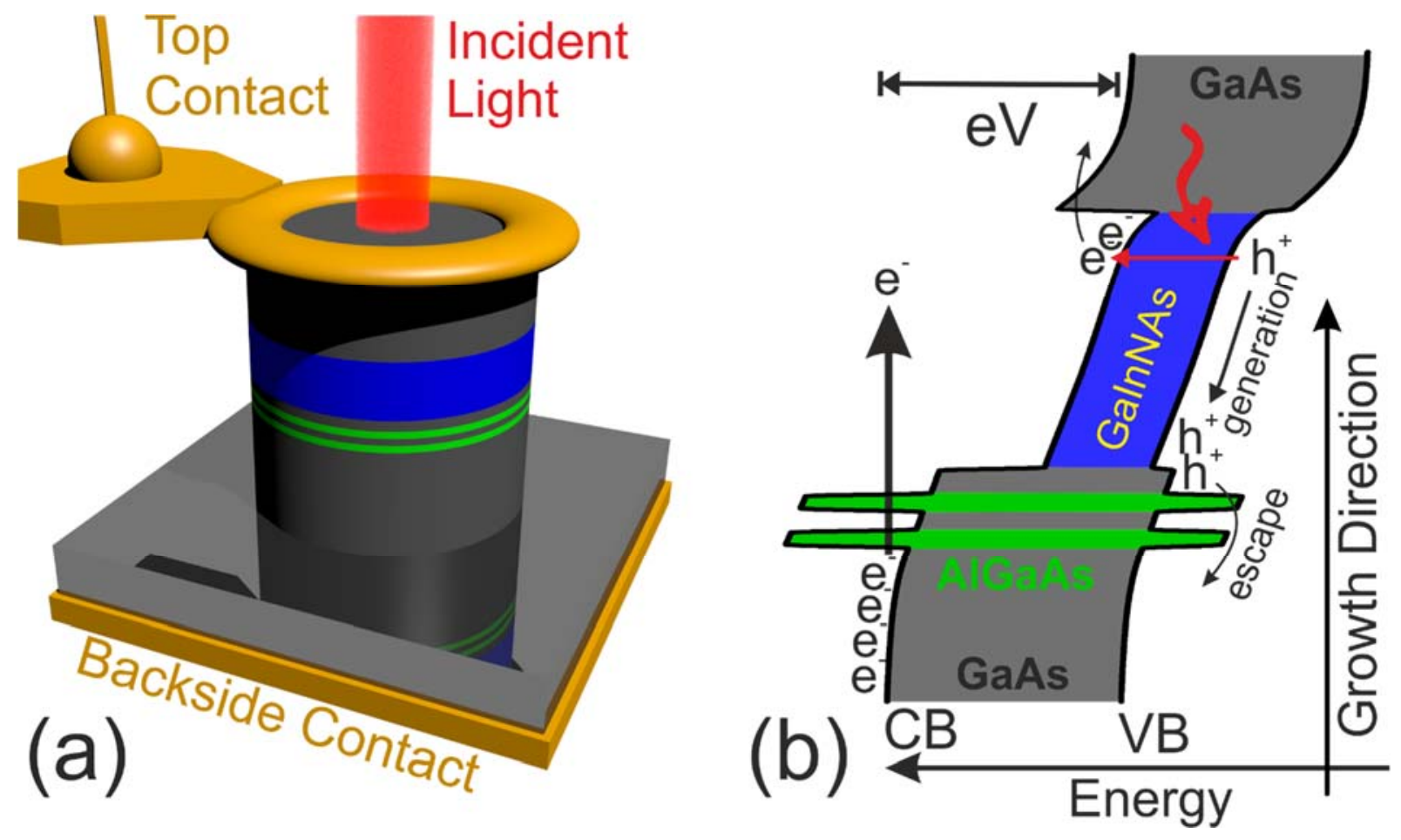

Figure 1: Sample design, working principle of the RTD photodetector. (a) Three dimensional sketch of the Resonant Tunneling Diode (RTD) photodetector. The structure is color-coded. Grey represents GaAs regions, whereas the GaInNAs absorption layer and the AIGaAs resonant tunneling barriers are blue and green, respectively. (b) Schematic conduction (CB) and valence band (VB) profile under an applied bias voltage. The CB and VB barrier heights of the AIGaAs barriers with respect to the GaAs $C B$ minimum and $V B$ maximum are $\triangle E_{C}=484 \mathrm{meV}$ and $\Delta E_{V}=305 \mathrm{meV}$, respectively. The VB offset at the GaAs/GaInNAs interface is approximately $\Delta E_{V \text { GaInNAs }} \cong 105 \mathrm{meV}$. When illuminated, electron-hole pairs are generated within the GalnNAs absorption layer. Photogenerated holes $\left(h^{+}\right)$drift towards the resonant tunneling structure (RTS), where they accumulate for a mean time before they escape again either by tunneling or thermionic emission. As a result of the localized positive charge, the local electrostatic potential is changed, which also changes the transmission probability of electrons (e-) through the RTS. 

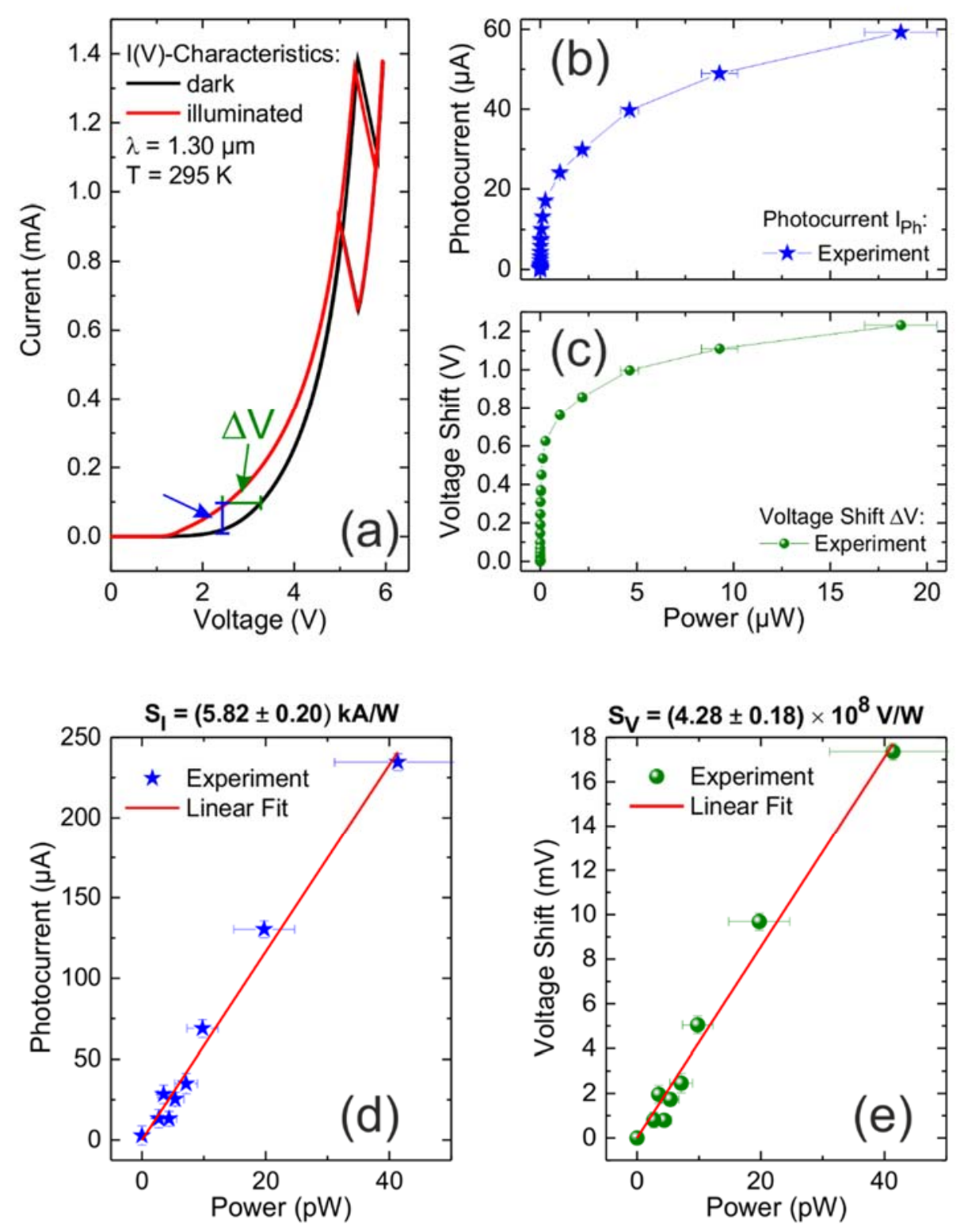

Figure 2: (a) I(V)-characteristics of the RTD photodetector in the dark (black line) and under illumination (red line). Under illumination, the I(V)-characteristic undergoes a voltage shift $\Delta V$ which gives rise to a photocurrent $I_{P h}$. (b) Photocurrent-power relation $I_{P h}(P)$ for illumination powers from $P=2.7 \mathrm{pW}$ up to $P=18.7 \mu \mathrm{W}$. (c) Corresponding photoinduced voltage shift $\Delta V(P)$ as a function of incident illumination power $P$. (d) Linear fit of $I_{P h}(P)$ at small powers. (e) Linear fit of $\Delta V(P)$ at small powers. 

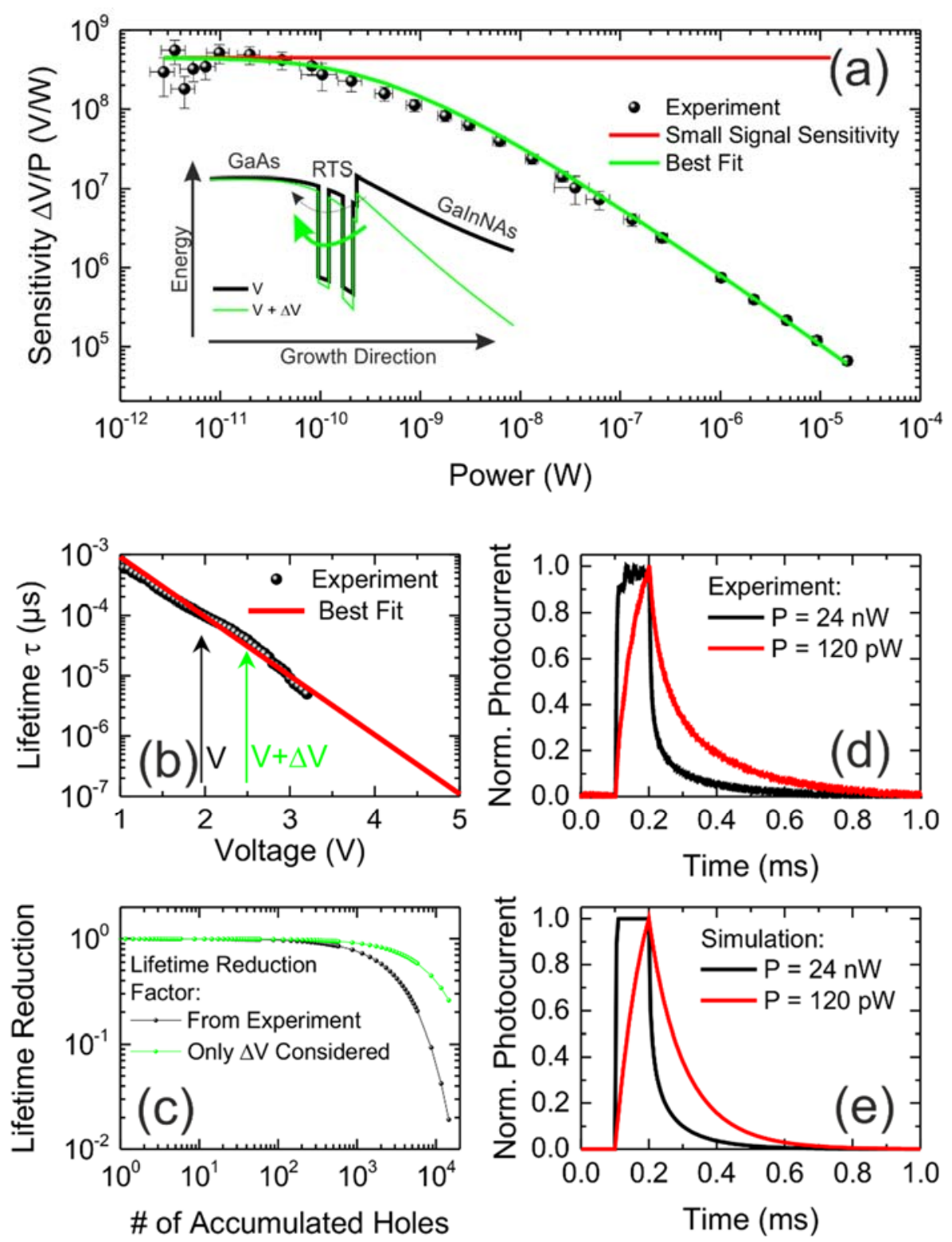

Figure 3: (a) Sensitivity of the photoinduced voltage shift $S_{V}(P)=\Delta V(P) / P$ versus incident illumination power $P$. Experimental data is shown as black spheres, the constant small signal sensitivity is indicated by the solid red line, and the best fit after Eq. (13) is shown as solid green line. The inset shows a sketch of the valence band under an applied bias voltage $V=V_{0}$ and under illumination, when voltage condition at the RTS corresponds to $V=V+\Delta V$. (b) Mean lifetime of photogenerated holes accumulated at the resonant tunneling structure versus bias voltage $V$. In case of small illumination powers, the lifetime of accumulated holes is given by $\tau(V)$, whereas for higher illumination powers the voltage condition at the RTS is changed and the mean lifetime is given by $\tau(V+\Delta V)$. (c) Reduction of the mean lifetime as a function of the increasing population of accumulated holes. (d) Normalized photocurrent time-traces for pulsed excitation with rectangular light pulses of $100 \mu$ s width and on-illumination powers of $P=24 \mathrm{nW}$ (black line) and $P=120 \mathrm{pW}$ (red line). (e) Simulation of the normalized photocurrent time-traces from part (d). 


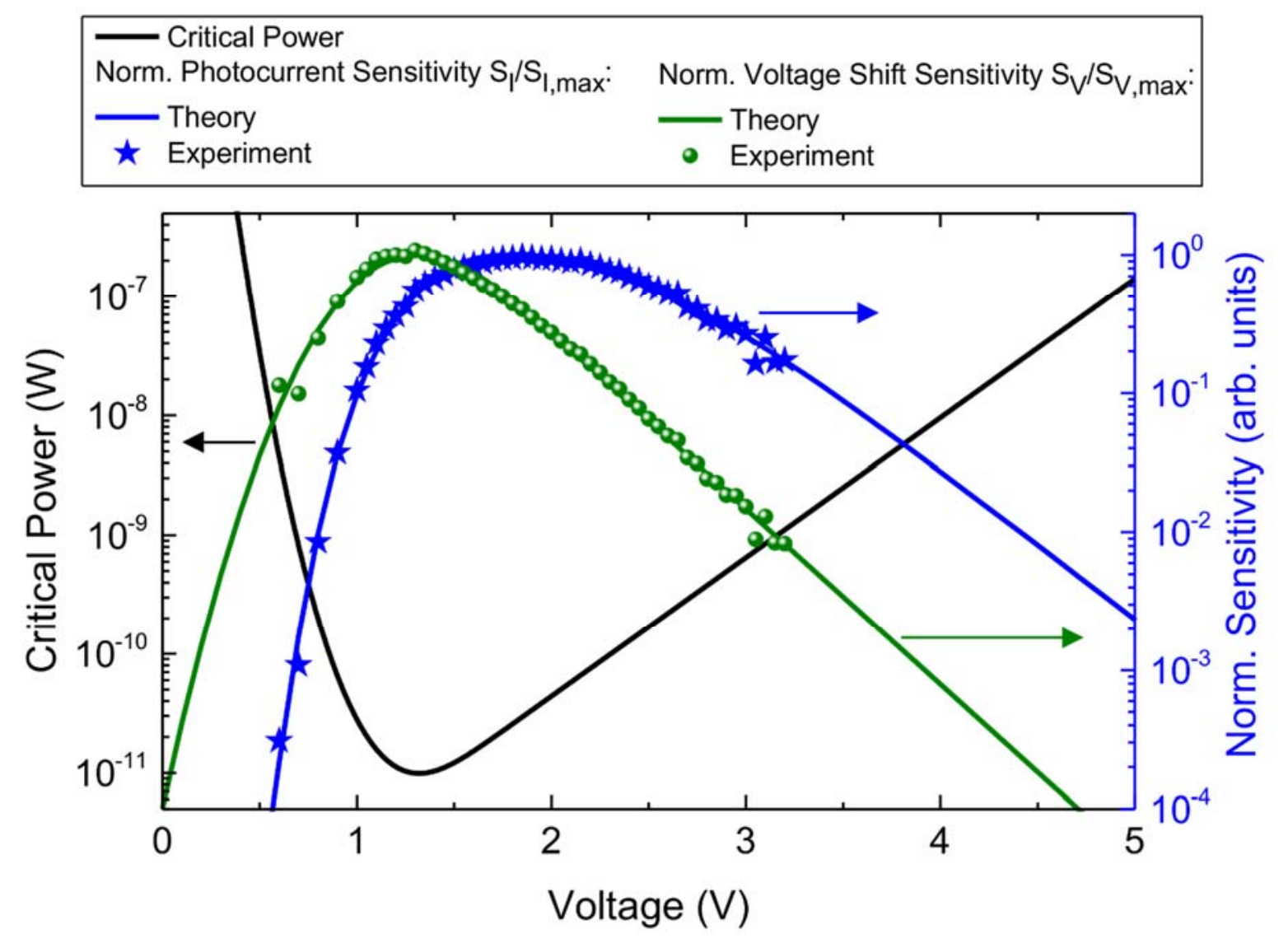

Figure 4: Critical illumination power $P_{\text {crit }}$ and normalized photocurrent and voltage shift sensitivities versus bias voltage $V$. The critical illumination power is defined as the power up to which the non-constant sensitivity has not deviated from the constant approximation by more than $10 \%$. The critical power has a minimum at $V=1.32 \mathrm{~V}$, which is where the voltage-shift sensitivity (determined by the product of $\tau(V)$ and $\eta(V)$ ) is maximal. To find the optimal working point, a trade-off has to be made between sensitivity and the critical power. 

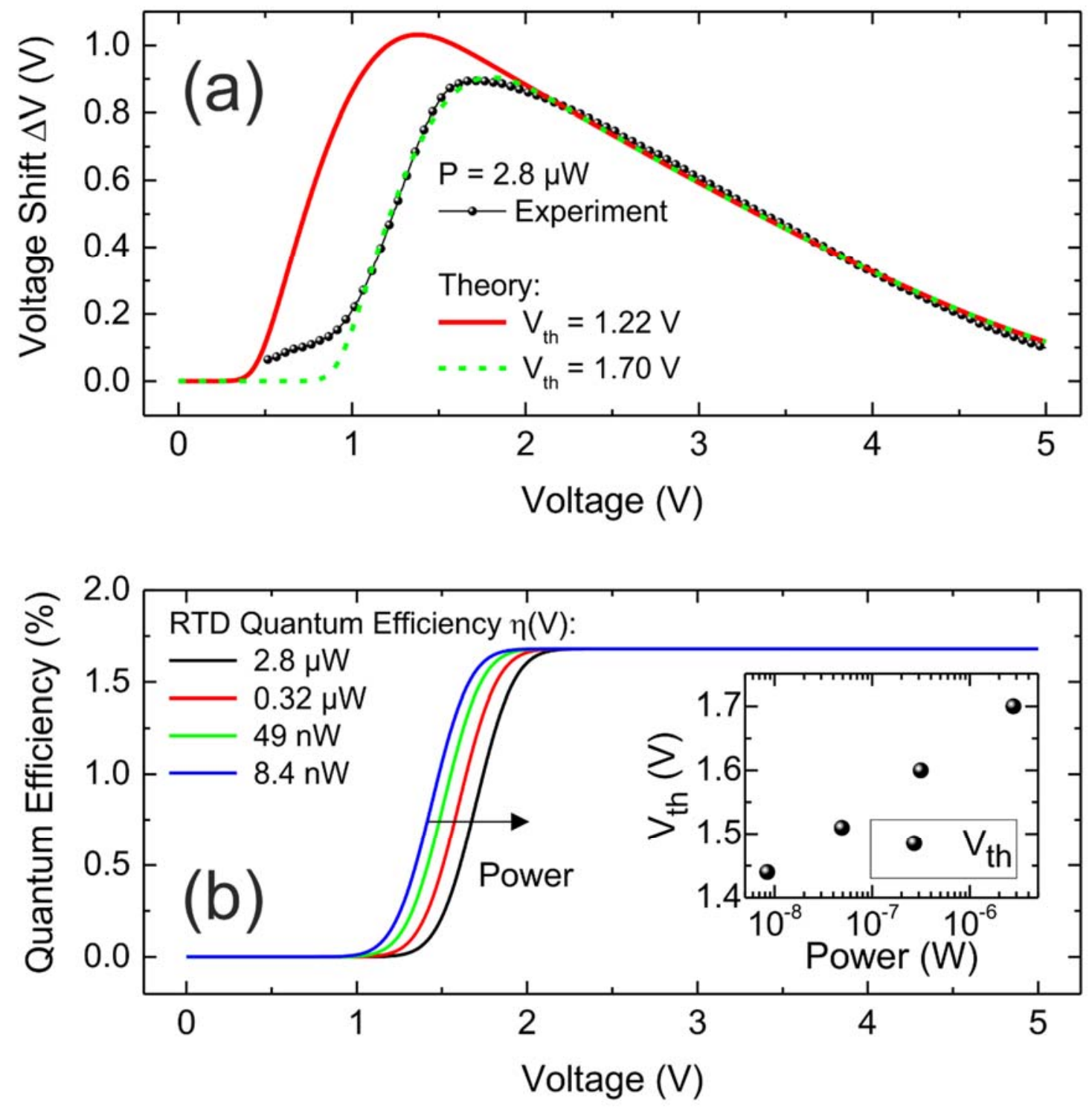

Figure 5: (a) Photoinduced voltage shift $\Delta V$ as a function of bias voltage for an illumination power of $P=2.8 \mu \mathrm{W}$. Experimental data is shown as black spheres. A theoretical calculation of $\Delta V(V)$ with the small signal RTD quantum efficiency threshold voltage of $V_{t h}=1.22 \mathrm{~V}$ is shown was solid red line. The best fit after Eq. (13) indicates a shift of $V_{t h}$ under illumination to $V_{\text {th }}=1.70 \mathrm{~V}$ and is shown as green dashed line. (b) RTD quantum efficiency $\eta(V)$ for various illumination powers from $P=8.4 \mathrm{nW}$ up to $2.8 \mu \mathrm{W}$. For increasing powers, the threshold voltage $V_{\text {th }}$ shifts to higher voltages. The inset shows the RTD quantum efficiency threshold voltage $V_{t h}$ versus incident illumination power $P$ on logarithmic scale. 

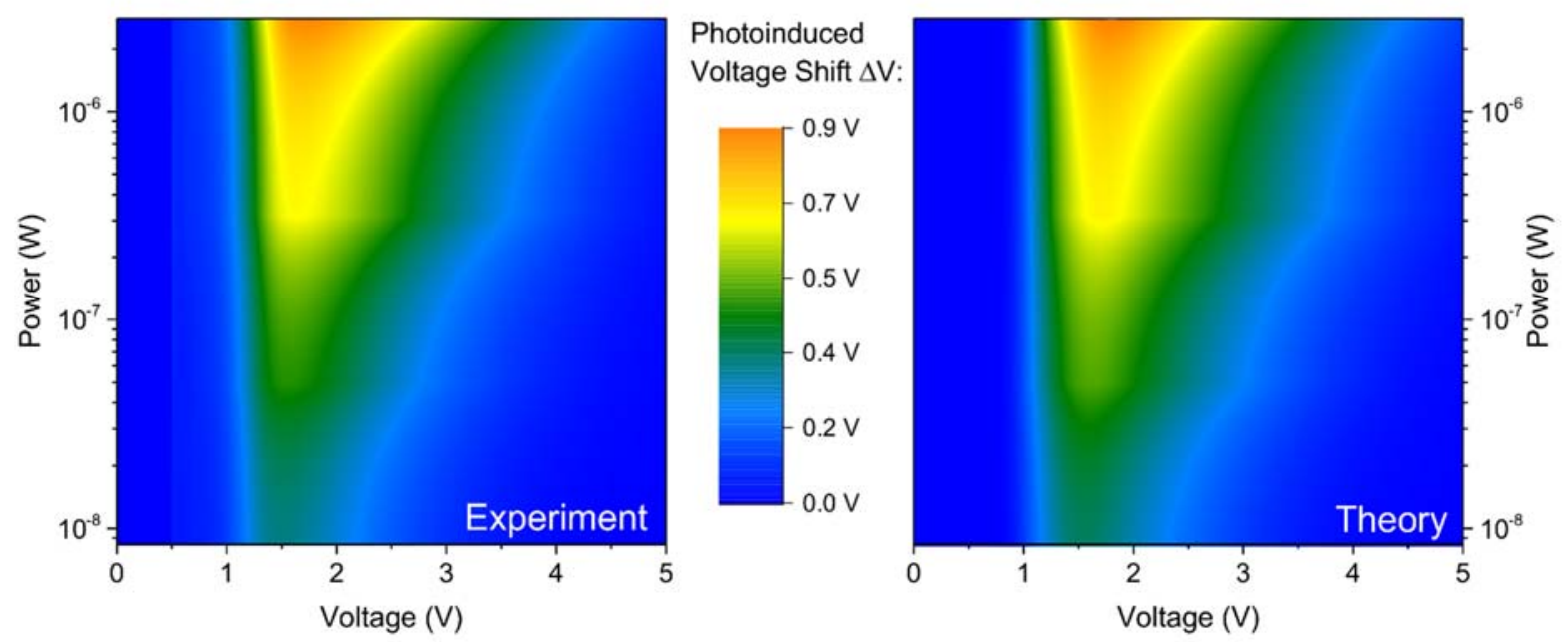

Figure 6: Colored contour plot of the photoinduced voltage shift $\Delta V$ as a function of bias voltage and illumination power. Orange represents the highest measured voltage shift with $\Delta V_{\max }=0.9 \mathrm{~V}$ and blue no voltage shift. Experiment (left-hand side) and theory (right-hand side) are in excellent agreement. 